\title{
Fibrinogen/Albumin Ratio as a Promising Marker for Predicting Survival in Pancreatic Neuroendocrine Neoplasms
}

This article was published in the following Dove Press journal: Cancer Management and Research

\author{
Shengming Deng ${ }^{1-4, *}$ \\ Zhiyao Fan ${ }^{\mathrm{I}-4, *}$ \\ Huanyu Xia ${ }^{\mathrm{I}-4, *}$ \\ Yitao Gong ${ }^{\text {-4 }}$ \\ Yunzhen Qian ${ }^{1-4}$ \\ Qiuyi Huang ${ }^{\text {I-4 }}$ \\ He Cheng ${ }^{I-4}$ \\ Kaizhou Jin ${ }^{1-4}$ \\ Zhiwen Xiao ${ }^{\mathrm{l}-4}$ \\ Guopei Luo ${ }^{\text {I-4 }}$ \\ Xianjun $\mathrm{Yu}^{\mathrm{l}-4}$ \\ Chen Liu ${ }^{\mathrm{I}-4}$ \\ 'Department of Pancreatic Surgery, \\ Fudan University Shanghai Cancer \\ Center, Shanghai, People's Republic of \\ China; ${ }^{2}$ Department of Oncology, \\ Shanghai Medical College, Fudan \\ University, Shanghai, People's Republic of \\ China; ${ }^{3}$ Shanghai Pancreatic Cancer \\ Institute, Shanghai, People's Republic of \\ China; ${ }^{4}$ Pancreatic Cancer Institute, \\ Fudan University, Shanghai, People's \\ Republic of China
}

*These authors contributed equally to this work
Background: The fibrinogen/albumin ratio (FAR) has been widely reported to be a possible biomarker for predicting prognosis in several types of tumors, but the prognostic value of the FAR in pancreatic neuroendocrine neoplasms (Pan-NENs) has not been systematically studied.

Patients and Methods: In total, 324 patients with Pan-NENs were recruited. The patients were divided into 2 subgroups according to the FAR cutoff value, and clinicopathological characteristics of the 2 subgroups were compared. Overall survival (OS) was the primary endpoint, and progression-free survival (PFS) was the secondary endpoint. The prognostic value of the FAR was analyzed in univariate and multivariate analyses.

Results: The optimal cutoff value for the FAR was calculated to be 0.08 for OS. The patients with a FAR $\geq 0.08$ had higher proportions of nonfunctioning tumors, Pan-NECs, grade 3 tumors, and stage IV tumors than those with a FAR $<0.08$. In the univariate analysis, a FAR $\geq 0.08$ was associated with poor OS (hazard ratio $(\mathrm{HR})=2.37, P<0.001$ ) and PFS (HR $=2.37, P<0.001)$. In the multivariate analysis, a FAR $\geq 0.08$ was an independent risk factor for poor OS $(\mathrm{HR}=4.70, P<0.001)$ and PFS $(\mathrm{HR}=1.80, P=0.006)$.

Conclusion: The pretreatment FAR, which includes fibrinogen and albumin, was a feasible and predictive biomarker for prognosis in patients with Pan-NENs. An elevated FAR, based on a cutoff value of 0.08 , was an independent risk factor for poor OS and PFS.

Keywords: pancreatic neuroendocrine neoplasms, survival, fibrinogen/albumin ratio

\section{Introduction}

Pancreatic neuroendocrine neoplasms (Pan-NENs) are a relatively rare type of pancreatic tumor that accounts for only $1-2 \%$ of all pancreatic malignancies, but the PanNEN incidence is rapidly increasing. ${ }^{1-3}$ In recent years, researchers have shown increasing interest in Pan-NENs. Pan-NENs are a heterogeneous disease with different prognoses in different subgroups. ${ }^{4,5}$ In addition to the existing tumor-node-metastasis (TNM) staging system, ${ }^{6}$ tumor grade, ${ }^{7}$ and differentiation status, ${ }^{8}$ there are a number of emerging markers for predicting the prognosis of patients with Pan-NENs, including the blood glucose level, ${ }^{9}$ peripheral blood neutrophil/lymphocyte ratio ${ }^{10}$ and some other peripheral blood test markers. However, no studies have explored the prognostic value of the fibrinogen/albumin ratio (FAR) in Pan-NENs.

Plasma fibrinogen is a soluble glycoprotein synthesized and secreted by liver cells and is an important protein involved in the processes of blood coagulation and hemostasis. ${ }^{11}$ In the presence of ionized calcium, fibrinogen is converted into fibrin
Correspondence: Chen Liu; Xianjun Yu Shanghai Pancreatic Cancer Institute, 270 Dong an Road, Shanghai 200032, People's Republic of China

Tel/Fax +86-2I-6403-I446

Email liuchen@fudanpci.org;

yuxianjun@fudanpci.org 
by thrombin. ${ }^{12}$ As an "acute-phase reactant", fibrinogen may play significant roles in various types of nonspecific stimuli, such as inflammation, pregnancy, and autoimmune diseases. ${ }^{13,14}$ Previous research has suggested that plasma fibrinogen may also play a pivotal role in the progression of various malignancies. ${ }^{15,16}$ Albumin is a circulating protein in the plasma and a nutritional indicator and can also participate in inflammation. ${ }^{17}$ Hypoalbuminemia is closely related to a low quality of life and has detrimental effects on therapeutic efficacy in cancer patients. ${ }^{18,19}$ Some studies have reported that the combination of fibrinogen and albumin can be used to predict the prognosis of cancer patients. $^{20,21}$ The baseline FAR was found to be an independent prognostic factor in patients with esophageal squamous cell carcinoma $^{20}$ soft tissue sarcoma, ${ }^{21}$ hepatocellular carcinoma, ${ }^{22}$ gastric cancer, ${ }^{23}$ and so on. In addition, researchers found that a high FAR was significantly associated with deeper tumor infiltration, lymph node metastasis and distant metastasis. ${ }^{24}$ To the best of our knowledge, no research has explored the prognostic value of the FAR in Pan-NENs.

Therefore, this study provides the first demonstration of an association between the FAR and survival in patients with Pan-NENs through a retrospective study involving 324 patients.

\section{Patients and Methods}

\section{Patients and the Study Design}

A retrospective follow-up study of Pan-NEN patients was conducted at the Shanghai Cancer Center, Fudan University, China, from January 2006 to December 2018. All enrolled patients were pathologically diagnosed based on histological or cytological evidence. According to the fifth edition of the World Health Organization (WHO) Classification of Tumors, Pan-NENs include well-differentiated pancreatic neuroendocrine tumors (Pan-NETs) and poorly differentiated neuroendocrine carcinomas (Pan-NECs). ${ }^{25}$ The exclusion criteria were as follows: (1) patients without follow-up data; (2) patients with a second malignant tumor or multiple primary malignant tumors; (3) patients lacking albumin and fibrinogen data; (4) patients with clinical evidence of infection or other inflammatory diseases (patients with an abnormal white blood cell count or signs or symptoms of infectious disease); and (5) patients with liver cirrhosis and other severe hepatic diseases. The study was approved by the Ethics Committee of the Shanghai Cancer Center of Fudan University. All patients in the study agreed to the use of their personal data for research purposes and provided written informed consent. The study was conducted according to the Declaration of Helsinki.

In total, 324 patients were recruited for our cohort. Clinicopathological patient data, including age, sex, tumor location, tumor grade, tumor differentiation, tumor stage, functioning tumors (yes or no), receipt of radical surgery (yes or no), and body mass index (BMI, $<18.5$ or $\geq 18.5$ ) were collected from medical records at the Shanghai Cancer Center. The tumor stage of each patient was based on the TNM staging for Pan-NENs established by the American Joint Committee on Cancer (AJCC), 8th edition. BMI was calculated based on the weight and height of patients on the day of admission, and according to guidelines of National Heart, Lung, and Blood Institute (NHLBI), we defined a BMI value less than 18.5 as low BMI. The levels of fibrinogen $(\mathrm{g} / \mathrm{L})$ and albumin $(\mathrm{g} / \mathrm{L})$ were obtained within 10 days prior to initial treatment.

Overall survival (OS), which was defined as the time from the first date of diagnosis to the date of death for any reason, was the primary endpoint. Patient OS data were ascertained through telephone follow-up. Progression-free survival (PFS), which was defined as the period from the onset of treatment to the observation of disease progression or death from any cause, was the secondary endpoint in this study. We evaluated patient disease progression according to the Response Evaluation Criteria in Solid Tumors (RECIST) (version 1.1) through imaging examinations (including computed tomography, magnetic resonance imaging, and positron emission tomography/computed tomography). ${ }^{26}$ Due to differences in tumor stages and treatment options, the frequency of imaging examination varied. Normally, patients undergoing imaging are evaluated every three months during the course of treatment. The frequency of reexamination for patients who have undergone radical surgery but do not need other treatment is gradually reduced.

\section{Statistical Methods}

Statistical analyses were performed using SPSS 24.0 software (SPSS, Inc., Chicago, IL) and R Studio version 3.6.1. Receiver operating characteristic (ROC) curves with Youden's Index correction were established to determine the optimal cutoff threshold of the FAR for OS. We utilized a Kaplan-Meier survival curve with a 95\% confidence interval (CI) for analyses of OS and PFS. Univariate and multivariate analyses were separately performed based on the Log rank test and the Cox proportional hazard model, respectively. Rank-sum tests, Pearson 
$\chi 2$ tests or Fisher's exact tests were used to compare clinicopathological characteristics between the groups. All $P$ values were two sided, and $P<0.05$ was considered statistically significant.

\section{Results}

\section{Clinical Characteristics of the Enrolled Patients with Pan-NENs}

A total of 324 patients were included in this study, and the mean follow-up time was 38.9 months. Selected clinical characteristics are shown in Table 1. Among the patients, 31 had Pan-NECs, and 293 had Pan-NETs; 69.4\% $(n=225)$ of the patients were younger than 60 years old. The prevalence of females in the study was $56.2 \%(\mathrm{n}=182)$. A total of $126(38.9 \%)$ patients had tumors located in the head of the pancreas, whereas $198(61.1 \%)$ had tumors located in the body or tail of the pancreas. The prevalence of functioning tumors in the study was $10.5 \%(\mathrm{n}=34)$. Of the entire patient cohort, $238(73.5 \%)$ patients underwent radical surgery, and 86 patients $(26.5 \%)$ did not. A total of 124 (39.1\%) patients had grade 1 Pan-NENs, 154 (48.6\%) had grade 2 Pan-NENs, and $39(12.3 \%)$ had grade 3 Pan-NENs. Moreover, 77 (23.8\%) patients had stage I Pan-NENs, 117 (36.1\%) had stage II Pan-NENs, 40 (12.3\%) had stage ш Pan-NENs, and 90 (27.8\%) had stage IV Pan-NENs.

\section{Correlations Between the FAR and Clinicopathological Factors}

The median FAR was 0.072 (range 0.036-0.170), and the optimal cutoff value for the FAR was calculated to be 0.08 for OS. Then, Pan-NEN patients were divided into 2 subgroups based on whether their FAR was lower than the cutoff value (0.08). Differences in clinicopathological characteristics between the subgroups are shown in Table 1. The prevalence of patients with a FAR $<0.08$ in the cohort was $70.4 \%(\mathrm{n}=228)$, and no significant differences in sex $(P=$ $0.146)$, location $(P=0.934)$ and BMI $(P=0.946)$ were found. The proportions of functioning tumors, patients younger than 60 years, and well-differentiated Pan-NETs in the group of Pan-NEN patients with a FAR $<0.08$ were higher than those in the group of patients with a FAR $\geq 0.08$. Notably, the distribution of populations with different tumor stages was significantly different between the two subgroups $(P=0.001)$. The proportion of patients with stage IV disease in the group of patients with a FAR $\geq 0.08$ was 1.99 times that of the group of patients with a FAR $<0.08(42.7 \%$ vs $21.5 \%)$. It is worth noting that $18(18.8 \%)$ patients with
Table I Clinicopathological Characteristics of All Patients

\begin{tabular}{|c|c|c|c|c|}
\hline Variables & $n$ & FAR $<0.08$ & $\begin{array}{l}\text { FAR } \geq \\
0.08\end{array}$ & $\boldsymbol{P}$ \\
\hline Total & 324 & 228 & 96 & \\
\hline Age (years), n (\%) & & & & 0.001 \\
\hline$<60$ & 225 & I7I (75.0) & $54(56.3)$ & \\
\hline$\geq 60$ & 99 & $57(25.0)$ & $42(43.7)$ & \\
\hline Sex, n (\%) & & & & 0.146 \\
\hline Female & 182 & $134(58.8)$ & $48(50.0)$ & \\
\hline Male & 142 & $94(4 \mid .2)$ & $48(50.0)$ & \\
\hline Location, n (\%) & & & & 0.934 \\
\hline Head & 126 & $89(39.0)$ & $37(38.5)$ & \\
\hline Body or tail & 198 & $137(61.0)$ & $59(61.5)$ & \\
\hline Functioning tumors, $\mathrm{n}(\%)$ & & & & $<0.001$ \\
\hline Yes & 34 & $33(13.9)$ & I (I.6) & \\
\hline No & 290 & $195(86.1)$ & $95(98.4)$ & \\
\hline Tumor grade, $\mathrm{n}(\%)^{\mathrm{a}}$ & & & & 0.032 \\
\hline Grade I & 124 & $94(42.5)$ & $30(31.3)$ & \\
\hline Grade 2 & 154 & $106(48.0)$ & $48(50.0)$ & \\
\hline Grade 3 & 39 & $21(9.5)$ & $18(18.8)$ & \\
\hline Tumor stage, n (\%) & & & & 0.001 \\
\hline I & 77 & $62(27.2)$ & $15(15.6)$ & \\
\hline II & 117 & $86(37.7)$ & $31(32.3)$ & \\
\hline III & 40 & $31(13.6)$ & $9(9.4)$ & \\
\hline IV & 90 & $49(21.5)$ & $4 \mathrm{I}(42.7)$ & \\
\hline $\begin{array}{l}\text { Tumor differentiation, } \\
\mathrm{n}(\%)\end{array}$ & & & & 0.046 \\
\hline Well & 293 & $211(92.5)$ & $82(85.4)$ & \\
\hline Poor & 31 & $17(7.5)$ & $14(14.6)$ & \\
\hline Radical surgery, n (\%) & & & & $<0.001$ \\
\hline Yes & 238 & I8I (79.4) & $57(59.4)$ & \\
\hline No & 86 & $47(20.6)$ & $39(40.6)$ & \\
\hline Body mass index, $\mathrm{n}(\%)^{\mathrm{b}}$ & & & & 0.946 \\
\hline$<18.5$ & 21 & $15(6.9 \%)$ & $6(6.7 \%)$ & \\
\hline$\geq 18.5$ & 287 & $\begin{array}{l}203 \\
(93.1 \%)\end{array}$ & 84 (93.3\%) & \\
\hline
\end{tabular}

Notes: a Data of tumor grade for 7 patients with were missing; ${ }^{b}$ data of body mass index for 16 patients were missing.

Abbreviation: FAR, fibrinogen/albumin ratio. 
a FAR $\geq 0.08$ and $21(9.5 \%)$ patients with a FAR $<0.08$ had tumors that were classified as grade 3 , and this difference was nearly two-fold. Furthermore, the majority of patients with a FAR $<0.08(\mathrm{n}=181,79.4 \%)$ underwent radical surgery, whereas only $57(59.4 \%)$ patients with a FAR $\geq 0.08$ underwent radical surgery $(P<0.001)$.

\section{Association Between the FAR and Survival in Patients with Pan-NENs}

At the end of the follow-up period, $14.5 \%$ of the participants $(\mathrm{n}=47)$ had died, and $36.9 \%(\mathrm{n}=116)$ had disease progression (10 participants had no data on recurrence). Briefly, a FAR $\geq 0.08$ was associated with poorer OS $(P<0.001$, Figure 1) and PFS $(P<0.001$, Figure 2$)$ than a FAR $<0.08$. The relationships between clinicopathological variables and OS are presented in Table 2 . In a univariate analysis, variables such as grade 2 tumors (hazard ratio $(\mathrm{HR})=3.92, P=$ $0.006)$, grade 3 tumors $(\mathrm{HR}=20.04, P<0.001)$, stage III tumors $(\mathrm{HR}=16.91, P=0.008)$, stage IV tumors $(\mathrm{HR}=$ $45.47, P<0.001)$, no receipt of radical surgery $(\mathrm{HR}=8.14$, $P<0.001)$, and a FAR $\geq 0.08(\mathrm{HR}=3.87, P<0.001)$ were risk factors for poor OS in patients with Pan-NENs. In a multivariate analysis, and a FAR $\geq 0.08$ (HR $=4.70, P<$ 0.001 ), along with variables such as tumors located in the head of the pancreas, grade 3 tumors and stage IV tumors, were independent risk factors for poor OS. Survival was significantly poorer for the patients with a FAR $\geq 0.08$ than those with a FAR $<0.08$, as shown in Figure $1(P<0.001)$.

Three hundred fourteen of the enrolled patients provided the necessary information for the determination of PFS. The relationships between clinicopathological variables and PFS are presented in Table 3. In a univariate analysis,

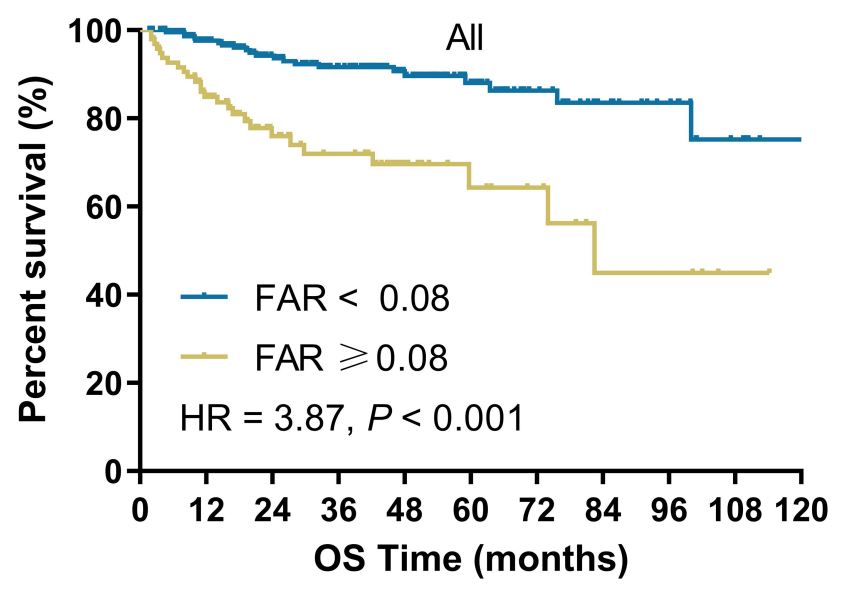

Figure I Kaplan-Meier plot of OS for all patients stratified into two groups: patients with a FAR $\geq 0.08$ and patients with a FAR $<0.08$.

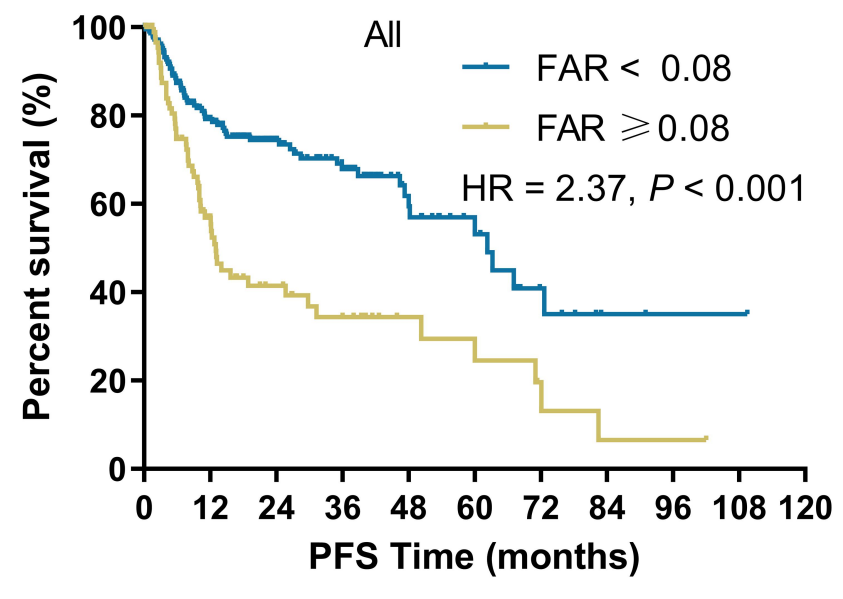

Figure 2 Kaplan-Meier plot of PFS for all patients stratified into two groups: patients with a FAR $\geq 0.08$ and patients with a FAR $<0.08$.

relatively poor PFS was significantly correlated with female sex $(H R=1.45, P=0.050)$, a tumor located in the body or tail of the pancreas $(\mathrm{HR}=1.48, P=0.048)$, functioning tumors $(\mathrm{HR}=2.63, P=0.021)$, grade 2 tumors $(\mathrm{HR}=3.61$, $P<0.001)$, grade 3 tumors $(\mathrm{HR}=10.66, P<0.001)$, stage II tumors $(\mathrm{HR}=6.06, P=0.014)$, stage III tumors $(\mathrm{HR}=$ $15.99, P<0.001)$, stage IV tumors $(\mathrm{HR}=71.57, P<0.001)$, no receipt of radical surgery $(\mathrm{HR}=8.93, P<0.001), \mathrm{BMI} \geq$ $18.5(\mathrm{HR}=2.30, P=0.013)$ and a FAR $\geq 0.08(\mathrm{HR}=2.37$, $P<0.001)$. A multivariate analysis revealed a FAR $\geq 0.08$ (HR $=1.73, P=0.007)$, along with grade 3 tumors and stage II, III \& IV tumors, were independent prognostic factors for unfavorable PFS. The median PFS time was 32.5 months in the patients with a FAR $\geq 0.08$ and 61.7 months in the patients with a FAR $<0.08$.

Moreover, we also analyzed the subgroup of patients who underwent radical surgical resection $(\mathrm{n}=238)$. Likewise, FAR value $>0.08$ was also associated with unfavorable overall survival $(P=0.042$, Figure 3$)$ and progression-free survival ( $\mathrm{P}=0.002$, Figure 4 ) for patients who underwent radical surgical resection.

\section{Discussion}

Pan-NENs are rare heterogeneous tumors, and their biological behavior is incompletely defined. ${ }^{4}$ With advancements in imaging technology, the detection rate of pancreatic neuroendocrine tumors is increasing in many parts of the world. ${ }^{27}$ This study attempted to explore the prognostic value of the FAR in Pan-NENs. Fibrinogen is not only an important component in the coagulation cascade but also an acutephase reactant associated with inflammation. ${ }^{13}$ Recently, there has been increasing evidence that fibrinogen plays important roles in tumor-related biological behaviors such 
Table 2 Univariate and Multivariate Analyses for the Overall Survival of All Patients According to Their Baseline FAR

\begin{tabular}{|c|c|c|c|c|c|c|}
\hline \multirow[t]{2}{*}{ Variables } & \multicolumn{3}{|c|}{ Univariate Analysis } & \multicolumn{3}{|c|}{ Multivariate Analysis } \\
\hline & HR & $95 \% \mathrm{Cl}$ & $P$ & HR & $95 \% \mathrm{Cl}$ & $P$ \\
\hline \multicolumn{7}{|l|}{ Age (years) } \\
\hline$<60$ & 1 & & & 1 & & \\
\hline$\geq 60$ & 0.99 & $0.53-1.85$ & 0.973 & 0.861 & $0.431-1.72$ & 0.671 \\
\hline \multicolumn{7}{|l|}{ Sex } \\
\hline Female & 1 & & & 1 & & \\
\hline Male & 0.64 & $0.36-1.13$ & 0.125 & 1.716 & $0.855-3.446$ & 0.129 \\
\hline \multicolumn{7}{|l|}{ Location } \\
\hline Head & 1 & & & I & & \\
\hline Body or tail & 0.82 & $0.46-1.47$ & 0.506 & 0.347 & $0.173-0.694$ & 0.003 \\
\hline \multicolumn{7}{|c|}{ Functioning tumors } \\
\hline Yes & 1 & & & 1 & & \\
\hline No & 0.15 & $0.02-1.08$ & 0.059 & 0.836 & $0.101-6.924$ & 0.868 \\
\hline \multicolumn{7}{|l|}{ Tumor grade } \\
\hline Grade I & 1 & & & 1 & & \\
\hline Grade 2 & 3.92 & $1.48-10.36$ & 0.006 & 5.498 & I.197-25.245 & 0.028 \\
\hline Grade 3 & 20.04 & $7.49-53.63$ & $<0.001$ & 16.377 & $3.313-80.953$ & 0.001 \\
\hline \multicolumn{7}{|l|}{ Tumor stage } \\
\hline I & I & & & I & & \\
\hline II & 2.80 & $0.33-24.02$ & 0.347 & 1.120 & $0.109-11.45$ & 0.924 \\
\hline III & 16.91 & $2.08-137.72$ & 0.008 & 3.612 & $0.376-34.682$ & 0.266 \\
\hline IV & 45.47 & $6.21-333.12$ & $<0.001$ & 7.792 & $0.759-80.04$ & 0.084 \\
\hline \multicolumn{7}{|l|}{ Radical surgery } \\
\hline Yes & 1 & & & 1 & & \\
\hline No & 8.14 & $4.43-14.97$ & $<0.001$ & 2.813 & $0.921-8.586$ & 0.069 \\
\hline \multicolumn{7}{|c|}{ Body mass index } \\
\hline \multicolumn{7}{|l|}{$<18.5$} \\
\hline$\geq 18.5$ & 0.443 & $0.173-1.131$ & 0.089 & 1.504 & $0.559-4.047$ & 0.419 \\
\hline \multicolumn{7}{|l|}{ FAR } \\
\hline$<0.08$ & 1 & & & I & & \\
\hline$\geq 0.08$ & 3.87 & $2.17-6.90$ & $<0.001$ & 4.56 & $2.306-9.567$ & $<0.001$ \\
\hline
\end{tabular}

Abbreviations: HR, hazard ratio; FAR, fibrinogen/albumin ratio.

as epithelial-mesenchymal transition, cell proliferation, and angiogenesis. ${ }^{28}$ Fibrinogen can promote tumor cell adhesion and invasion by providing a stable framework for the tumor extracellular matrix. ${ }^{29}$ In addition, an important link has been established between this hemostatic factor and innate immunity. Platelets and fibrinogen can increase the metastatic 
Table 3 Univariate and Multivariate Analyses for the Progression-Free Survival of All Patients According to Their Baseline FAR

\begin{tabular}{|c|c|c|c|c|c|c|}
\hline \multirow[t]{2}{*}{ Variables } & \multicolumn{3}{|c|}{ Univariate Analysis } & \multicolumn{3}{|c|}{ Multivariate Analysis } \\
\hline & HR & $95 \% \mathrm{Cl}$ & $P$ & HR & $95 \% \mathrm{Cl}$ & $P$ \\
\hline \multicolumn{7}{|l|}{ Age (years) } \\
\hline$<60$ & 1 & & & 1 & & \\
\hline$\geq 60$ & 1.02 & $0.69-1.52$ & 0.916 & 0.677 & $0.436-1.052$ & 0.083 \\
\hline \multicolumn{7}{|l|}{ Sex } \\
\hline Female & 1 & & & 1 & & \\
\hline Male & 0.69 & $0.48-1.00$ & 0.050 & 0.743 & $0.493-1.121$ & 0.157 \\
\hline \multicolumn{7}{|l|}{ Location } \\
\hline Head & 1 & & & I & & \\
\hline Body or tail & 1.48 & $01.00-2.19$ & 0.048 & 1.090 & $0.705-1.685$ & 0.700 \\
\hline \multicolumn{7}{|c|}{ Functioning tumors } \\
\hline Yes & 1 & & & I & & \\
\hline No & 0.38 & $0.17-0.86$ & 0.021 & 0.876 & $0.537-2.148$ & 0.772 \\
\hline \multicolumn{7}{|l|}{ Tumor grade } \\
\hline Grade I & 1 & & & I & & \\
\hline Grade 2 & 3.61 & $2.12-6.15$ & $<0.001$ & 1.491 & $0.789-2.818$ & 0.219 \\
\hline Grade 3 & 10.66 & $5.87-19.37$ & $<0.001$ & 1.920 & $0.919-4.011$ & 0.083 \\
\hline \multicolumn{7}{|l|}{ Tumor stage } \\
\hline I & I & & & I & & \\
\hline II & 6.06 & $1.43-25.62$ & 0.014 & 4.969 & $1.138-21.704$ & 0.033 \\
\hline III & 15.99 & $3.68-69.59$ & $<0.001$ & 7.579 & $1.613-35.613$ & 0.010 \\
\hline IV & 71.57 & $17.45-293.53$ & $<0.001$ & 27.168 & $5.524-|33.62|$ & $<0.001$ \\
\hline \multicolumn{7}{|l|}{ Radical surgery } \\
\hline Yes & 1 & & & 1 & & \\
\hline No & 8.93 & $6.02-13.24$ & $<0.001$ & 1.827 & $0.88-6.07$ & 0.072 \\
\hline \multicolumn{7}{|c|}{ Body mass index } \\
\hline \multicolumn{7}{|l|}{$<18.5$} \\
\hline$\geq 18.5$ & 0.434 & $0.225-0.837$ & 0.013 & 0.796 & $0.399-1.586$ & 0.516 \\
\hline \multicolumn{7}{|l|}{ FAR } \\
\hline$<0.08$ & I & & & I & & \\
\hline$\geq 0.08$ & 2.37 & $1.64-3.42$ & $<0.001$ & 1.803 & $1.185-2.744$ & 0.006 \\
\hline
\end{tabular}

Abbreviations: $H R$, hazard ratio; FAR, fibrinogen/albumin ratio.

potential of tumors by preventing natural killer cells from eliminating tumor cells. ${ }^{30}$ Moreover, there is a close relationship between fibrinogen and systemic inflammation. Many studies have reported that plasma fibrinogen levels are positively correlated with many inflammation scores, such as the neutrophil/lymphocyte ratio, platelet/lymphocyte ratio and 


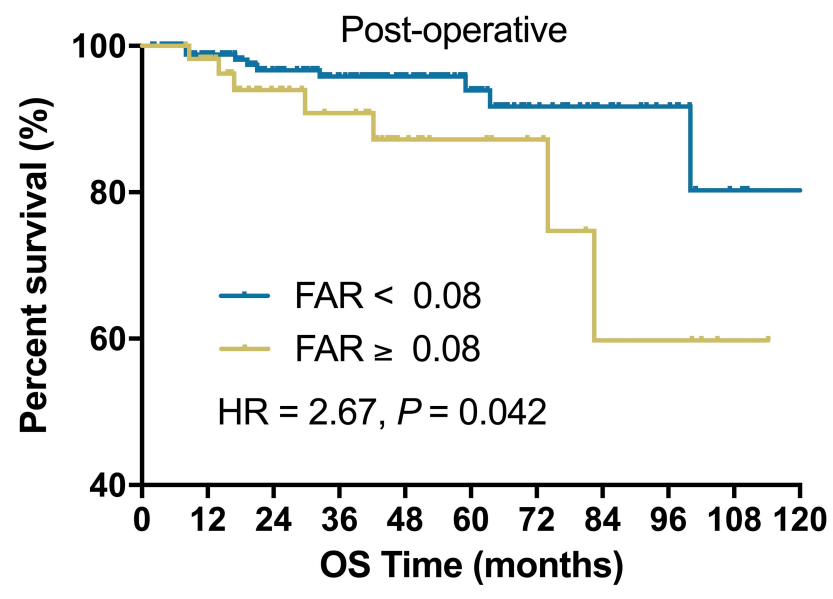

Figure 3 Kaplan-Meier plot of OS for patients without radical surgical resection stratified into two groups: patients with a FAR $\geq 0.08$ and patients with a FAR $<0.08$.

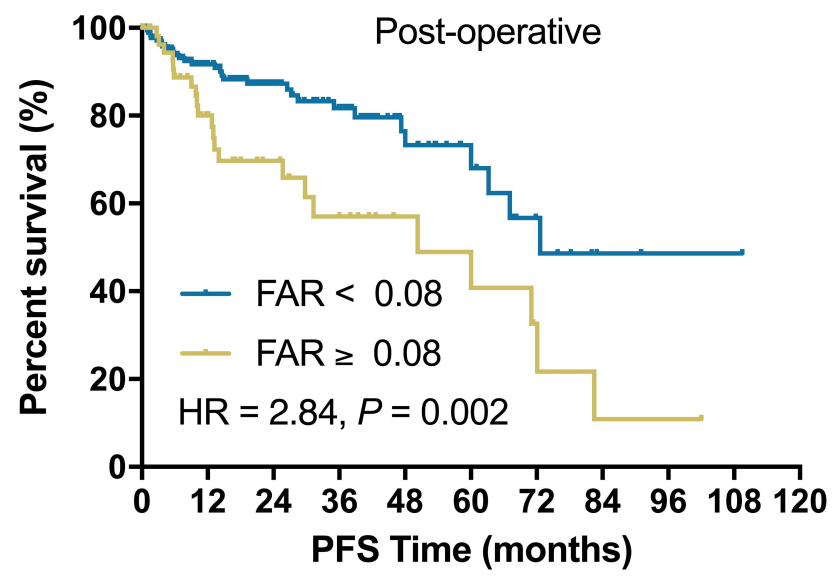

Figure 4 Kaplan-Meier plot of PFS for patients without radical surgical resection stratified into two groups: patients with a FAR $\geq 0.08$ and patients with a FAR $<0.08$.

lymphocyte/monocyte ratio. ${ }^{23,31}$ Fibrinogen can also induce the synthesis of interleukin 6 to promote tumor progression. ${ }^{32}$

Albumin is the most abundant circulating protein in the plasma and is not only a nutritional indicator but can also be involved in the inflammatory response. ${ }^{17}$ When the body is in an inflamed or malnourished state, albumin synthesis is significantly reduced. ${ }^{33,34}$ Hypoalbuminemia may cause impairment in the immune function of tumor patients, reduce therapeutic effects, and increase mortality. ${ }^{35}$ Additionally, many studies have shown that hypoalbuminemia is an independent risk factor for a poor prognosis in many cancers. ${ }^{36}$ The FAR can potentially represent the inflammatory status and nutritional status of the body. Many studies have shown that an elevated FAR is closely related to a poor prognosis in various cancers. ${ }^{37}$
To the best of our knowledge, our study is the first to report on the prognostic role of the FAR in Pan-NENs.

In this study, a total of 324 patients with Pan-NENs were enrolled. The cutoff value for the FAR was calculated to be 0.08 for OS, and the patients were divided into two groups according to whether their FAR was lower than 0.08. Surprisingly, the two groups presented different characteristics: the patients with a FAR $\geq 0.08$ had higher proportions of nonfunctioning tumors, Pan-NECs, grade 3 tumors, and stage IV tumors than the patients with a FAR $<0.08$. This suggests that patients with a relatively high FAR may be more prone to invasion and metastasis. Additionally, the patients with a FAR $\geq 0.08$ at the time of study enrollment exhibited worse OS and PFS than the patients with a FAR $<$ 0.08. In univariate and multivariate analyses, a FAR $\geq 0.08$ was associated with poor OS and PFS. The median PFS time in the patients with a FAR $<0.08$ was 1.90 times that of the patients with a FAR $\geq 0.08$. An elevated FAR is an independent risk factor for a poor prognosis in patients with Pan-NENs.

There are also some limitations to this research. As a retrospective, single-center study, these results require validation through multiple neutral prospective studies. Second, differences in patient treatments might lead to confusion with regard to the results. In addition, due to an insufficient follow-up time period, the median survival time could not be calculated.

Despite these limitations, our study is the first to show that the pretreatment FAR, which includes both fibrinogen and albumin, is a feasible and predictive biomarker for prognosis in patients with Pan-NENs. An elevated FAR, as defined by a cutoff value of 0.08 , was an independent risk factor for poor OS and PFS.

\section{Acknowledgment}

This work was supported by the National Natural Science Foundation of China (grant numbers 81625016, 81871940, 81902417), the Scientific Innovation Project of Shanghai Education Committee (2019-01-07-00-07-E00057), Clinical and Scientific Innovation Project of Shanghai Hospital Development Center (SHDC12018109), the Shanghai Natural Science Foundation (grant number 17ZR1406300), the Shanghai Cancer Center Foundation for Distinguished Young Scholars (grant number YJJQ201803), and the Fudan University Personalized Project for "Double Top" Original Research (grant number XM03190633). 


\section{Disclosure}

The authors report no conflicts of interest for this work.

\section{References}

1. Yao J, Hassan M, Dagohoy A. C, et al. One hundred years after "carcinoid": epidemiology of and prognostic factors for neuroendocrine tumors in 35,825 cases in the United States. J Clin Oncol. 2008;26:3063. doi:10.1200/JCO.2007.15.4377

2. Ito T, Igarashi $\mathrm{H}$, Nakamura $\mathrm{K}$, et al. Epidemiological trends of pancreatic and gastrointestinal neuroendocrine tumors in Japan: a nationwide survey analysis. $J$ Gastroenterol. 2015;50(1):58-64. doi:10.1007/s00535-014-0934-2

3. Halfdanarson TR, Rabe KG, Rubin J, et al. Pancreatic neuroendocrine tumors (PNETs): incidence, prognosis and recent trend toward improved survival. Ann Oncol. 2008;19(10):1727-1733. doi:10.1093/ annonc/mdn351

4. Ohmoto A, Rokutan H, Yachida S. Pancreatic Neuroendocrine Neoplasms: basic Biology, Current Treatment Strategies and Prospects for the Future. Int J Mol Sci. 2017;18(1):143. doi:10.3390/ijms18010143

5. Sun J. Pancreatic neuroendocrine tumors. Intractable Rare Dis Res. 2017;6(1):21-28. doi:10.5582/irdr.2017.01007

6. Luo G, Javed A, Strosberg JR, et al. Modified Staging Classification for Pancreatic Neuroendocrine Tumors on the Basis of the American Joint Committee on Cancer and European Neuroendocrine Tumor Society Systems. J Clin Oncol. 2017;35(3):274-280. doi:10.1200/ JCO.2016.67.8193

7. Botling J, Lamarca A, Bajic D, et al. High-Grade Progression Confers Poor Survival in Pancreatic Neuroendocrine Tumors.. Neuroendocrinology. 2020;110(11-12):891-898. doi:10.1159/ 000504392

8. Milione M, Maisonneuve P, Spada F, et al. The Clinicopathologic Heterogeneity of Grade 3 Gastroenteropancreatic Neuroendocrine Neoplasms: morphological Differentiation and Proliferation Identify Different Prognostic Categories. Neuroendocrinology. 2017;104 (1):85-93. doi:10.1159/000445165

9. Sandini M, Strobel O, Hank T, et al. Pre-operative dysglycemia is associated with decreased survival in patients with pancreatic neuroendocrine neoplasms. Surgery. 2020;167(3):575-580. doi:10.1016/ j.surg.2019.11.007

10. Tong Z, Liu L, Zheng Y, et al. Predictive value of preoperative peripheral blood neutrophil/lymphocyte ratio for lymph node metastasis in patients of resectable pancreatic neuroendocrine tumors: a nomogram-based study. World J Surg Oncol. 2017;15(1):108. doi:10.1186/s12957-017-1169-5

11. Weisel JW, Litvinov RI. Fibrin Formation, Structure and Properties. Subcell Biochem. 2017;82:405-456.

12. Halper J, Kjaer M. Basic components of connective tissues and extracellular matrix: elastin, fibrillin, fibulins, fibrinogen, fibronectin, laminin, tenascins and thrombospondins. Adv Exp Med Biol. 2014;802:31-47.

13. Luyendyk JP, Schoenecker JG, Flick MJ. The multifaceted role of fibrinogen in tissue injury and inflammation. Blood. 2019;133 (6):511-520. doi:10.1182/blood-2018-07-818211

14. Hoppe B, Häupl T, Skapenko A, et al. Fibrinogen and factor XIII A-subunit genotypes interactively influence C-reactive protein levels during inflammation. Ann Rheum Dis. 2012;71(7):1163-1169. doi:10.1136/annrheumdis-2011-200738

15. Wang M, Zhang G, Zhang Y, et al. Fibrinogen Alpha Chain Knockout Promotes Tumor Growth and Metastasis through Integrin-AKT Signaling Pathway in Lung Cancer. Mol Cancer Res. 2020.

16. Adams GN, Rosenfeldt L, Frederick M, et al. Colon Cancer Growth and Dissemination Relies upon Thrombin, Stromal PAR-1, and Fibrinogen. Cancer Res. 2015;75(19):4235-4243. doi:10.1158/00085472.CAN-15-0964
17. Caraceni P, Tufoni M, Bonavita ME. Clinical use of albumin.. Blood Transfus. 2013;11 Suppl 4:S18-S25. doi:10.2450/2013.005s

18. Rogers PCJ. Nutritional status as a prognostic indicator for pediatric malignancies. J Clin Oncol. 2014;32(13):1293-1294. doi:10.1200/ JCO.2014.55.0616

19. Fan Z, Fan K, Gong Y, et al. $<\mathrm{p}>$ The CRP/Albumin Ratio Predicts Survival And Monitors Chemotherapeutic Effectiveness In Patients With Advanced Pancreatic Cancer. Cancer Manag Res. 2019;11:8781-8788. doi:10.2147/CMAR.S211363

20. Tan Z, Zhang M, Han Q, et al. A novel blood tool of cancer prognosis in esophageal squamous cell carcinoma: the Fibrinogen/ Albumin Ratio. J Cancer. 2017;8(6):1025-1029. doi:10.7150/ jca. 16491

21. Liang Y, Wang W, Que Y, et al. Prognostic value of the fibrinogen/albumin ratio (FAR) in patients with operable soft tissue sarcoma. BMC Cancer. 2018;18(1):942. doi:10.1186/s12885018-4856-x

22. Xu Q, Yan Y, Gu S, et al. A Novel Inflammation-Based Prognostic Score: the Fibrinogen/Albumin Ratio Predicts Prognoses of Patients after Curative Resection for Hepatocellular Carcinoma. J Immunol Res. 2018;2018:4925498. doi:10.1155/2018/4925498

23. Zhang J, Ding Y, Wang W, et al. Combining the Fibrinogen/Albumin Ratio and Systemic Inflammation Response Index Predicts Survival in Resectable Gastric Cancer. Gastroenterol Res Pract. 2020;2020:3207345. doi:10.1155/2020/3207345

24. Zhang Y, Xiao G. $<\mathrm{p}>$ Prognostic significance of the ratio of fibrinogen and albumin in human malignancies: a meta-analysis. Cancer Manag Res. 2019;11:3381-3393. doi:10.2147/CMAR. S198419

25. Rindi G, Klimstra DS, Abedi-Ardekani B, et al. A common classification framework for neuroendocrine neoplasms: an International Agency for Research on Cancer (IARC) and World Health Organization (WHO) expert consensus proposal. Mod Pathol. 2018;31(12):1770-1786. doi:10.1038/s41379-018-0110-y

26. Nishino M, Jagannathan JP, Ramaiya NH, et al. Revised RECIST guideline version 1.1: what oncologists want to know and what radiologists need to know. AJR Am J Roentgenol. 2010;195 (2):281-289. doi:10.2214/AJR.09.4110

27. Baur AD, Pavel M, Prasad V, et al. Diagnostic imaging of pancreatic neuroendocrine neoplasms (pNEN): tumor detection, staging, prognosis, and response to treatment. Acta Radiol. 2016;57(3):260-270. doi: $10.1177 / 0284185115579932$

28. Zhang X, Wang F, Huang Y, et al. $<\mathrm{p}>\mathrm{FGG}$ promotes migration and invasion in hepatocellular carcinoma cells through activating epithelial to mesenchymal transition. Cancer Manag Res. 2019;11:1653-1665. doi:10.2147/CMAR.S188248

29. Sahni A, Simpson-haidaris PJ, Sahni SK, et al. Fibrinogen synthesized by cancer cells augments the proliferative effect of fibroblast growth factor-2 (FGF-2). J Thromb Haemost. 2007;6(1):176-183. doi:10.1111/j.1538-7836.2007.02808.x

30. Palumbo JS, Talmage KE, Massari JV, et al. Platelets and fibrin(ogen) increase metastatic potential by impeding natural killer cell-mediated elimination of tumor cells. Blood. 2005;105(1):178-185. doi:10. 1182/blood-2004-06-2272

31. Zheng Y, Wu C, Yan $\mathrm{H}$, et al. Prognostic value of combined preoperative fibrinogen-albumin ratio and platelet-lymphocyte ratio score in patients with breast cancer: A prognostic nomogram study. Clin Chim Acta. 2020;506:110-121. doi:10.1016/j. cca.2020.03.011

32. Ridker PM, Howard CP, Walter V, et al. Effects of interleukin-1 $\beta$ inhibition with canakinumab on hemoglobin A1c, lipids, C-reactive protein, interleukin- 6 , and fibrinogen: a phase IIb randomized, placebo-controlled trial. Circulation. 2012;126(23):2739-2748. doi:10.1161/CIRCULATIONAHA.112.122556

33. Hülshoff A, Schricker T, Elgendy H, et al. Albumin synthesis in surgical patients. Nutrition. 2013;29(5):703-707. doi:10.1016/j.nut.2012.10.014 
34. Baracos VE. Cancer-associated malnutrition. Eur $J$ Clin Nutr. 2018;72(9):1255-1259. doi:10.1038/s41430-018-0245-4

35. Hu W-H, Eisenstein S, Parry L, et al. Preoperative malnutrition with mild hypoalbuminemia associated with postoperative mortality and morbidity of colorectal cancer: a propensity score matching study. Nutr J. 2019;18(1):33. doi:10.1186/s12937-019-0458-y

36. Lim WS, Roh J-L, Kim S-B, et al. Pretreatment albumin level predicts survival in head and neck squamous cell carcinoma Laryngoscope. 2017;127(12):E437-E442. doi:10.1002/lary.26691
37. Sun Y, Zhang Y, Huang Z, et al. Combination of Preoperative Plasma Fibrinogen and Neutrophil-to-Lymphocyte Ratio (the F-NLR Score) as a Prognostic Marker of Locally Advanced Rectal Cancer Following Preoperative Chemoradiotherapy. World J Surg. 2020;44 (6):1975-1984. doi:10.1007/s00268-020-05407-3

\section{Publish your work in this journal}

Cancer Management and Research is an international, peer-reviewed open access journal focusing on cancer research and the optimal use of preventative and integrated treatment interventions to achieve improved outcomes, enhanced survival and quality of life for the cancer patient.
The manuscript management system is completely online and includes a very quick and fair peer-review system, which is all easy to use. Visit http://www.dovepress.com/testimonials.php to read real quotes from published authors. 